\title{
The Value of C-Reactive Protein as a Predictor of Vasospasm and Poor Outcome in Spontaneous Subarachnoid Hemorrhage
}

\author{
MOHAMED ELMALLAWANY, M.D.; AHMED ATTIA, M.Sc.; AHMED EL-FIKI, M.D.; \\ MOHAMED TAREK EL FAR, M.D. and AHMED ZATER, M.D.
}

The Department of Neurosurgery, Faculty of Medicine, Cairo University

\begin{abstract}
Background: Rupture of an intracranial aneurysm carries a high risk of disability or death. Cerebral vasospasm remains the most troublesome complication of acute Subarachinoidhaemorraghe (SAH). Activation of the systemic immune response, manifested by increased levels of circulating cytokines, is believed to have a significant role in the pathogenesis of cerebrovascular vasospasm.
\end{abstract}

Aim of Study: The aim of this study is to correlatebetween level of CRP and incidence of vasospasm in spontaneous S.A.H, and being an independent predictor for prognosis.

Patients and Methods: Cross sectional study with stratified random sample of 30 patients who admitted in Intensive Care Unit, Neurosurgery Department, Emergency and Trauma Unit at Kasr Al-Ainy, Cairo University Hospitals, from September 2018 till April 2019 diagnosed with spontaneous Subarachnoid hemorrhage and screened for CRP.

Results: Out of the 30 included in the study 10 patients $(33.3 \%)$ diagnosed to have anterior communicating artery aneurysm; 2 patients $(6.7 \%)$ have basilar tip aneurysm, 2 patients $(6.7 \%)$ have posterior communicating artery aneurysm, 2 patients $(6.7 \%)$ have posterior cerebral aneurysm, and 1 patient $(1.9 \%)$ have middle cerebral artery aneurysm, 2 patient have AVM, 11 patient have no underlying pathology in the conventional 4 vessels angiography.

Overall age ranged from 21 years to 80 years with a mean age 53.19 patients $(63.3 \%)$ were males and 11 patients $(36.7 \%)$ were females.

Out from 30 patients, 14 patients $(46.7 \%)$ had vasospasm, and $16(53.3 \%)$ patients didn't have vasospasm, out of 14 patients who developed vasospasm, 13 patients $(92 \%)$ initially had high serum CRP level above $50 \mathrm{mg} / \mathrm{L}$, and all patient who had rising CRP level (double to triple folds) had poor outcome.

Conclusion: Vasospasm is a serious event complicating spontaneous aneurysmal subarachnoid hemorrhage, it could be diagnosed clinically or radiologically, serum CRP level is an indicator of cerebral vasospasm following aneurysmal subarachnoid hemorrhage and rising serum CRP level is an independent predictor for poor outcome.

Correspondence to: Dr. Mohamed Elmallawany, The Department of Neurosurgery, Faculty of Medicine, Cairo University
Key Words: Spontaneous Subarachnoid hemorrhage - Cerebral vasospasm - C-reactive protein.

\section{Introduction}

RUPTURE of an intracranial aneurysm carries a high risk of disability ordeath [1].

A previous international study stated that of the patients who had survived the initial ictus, 33\% of the patients were rendered either severely disabled, vegetative, or dead after aneurysmal Subarachnoid Hemorrhage (SAH) [2].

Cerebral vasospasm continues to be the most troublesome complication of subarachnoid hemorrhage. It is associated with avery high morbidity and mortality, even after successful treatment of the ruptured aneurysm [1].

The incidence of vasospasm is estimated to be $70 \%$ based onconventional angiography, and in $20-30 \%$ of the patients, vasospasm is responsible for the development of a clinical vasospasm or what so called Delayed Ischemic Neurological Deficit (DIND) [2].

Activation of the systemic immune response, manifested by increased levels of circulating cytokines, is believed to have a significant role in the pathogenesis of cerebrovascular vasospasm [4]

Multiple inflammatory reactions are involved in the pathogenesis of several vascular diseases [5].

The levels of C-Reactive Protein (CRP), which is a highly sensitive inflammatory marker, are associated with the severity and outcome of theischemic stroke, coronary artery disease and atherosclerosis [6] 
CRP had also been investigated in patients diagnosed with aneurysmal SAH [5]. Intracranial hypertension, a complication which can follow aneurysmal SAH, is linked to high interleukin-6 and CRP levels in both the brain and systemic circulation, suggesting a significant role of the inflammatory processes in the pathogenesis of complications post-aneurysmal SAH [7].

Several reports showed that an elevated CRP level in the serum or the cerebrospinal fluid was correlated with a poor outcome and/or vasospasm after SAH due to a ruptured aneurysm [8]

In contrast, another study declared that, patients showing vasospasm or poor outcome had a higher CRP values from day of admission to the ninth day postadmission [9].

Other study showed that CRP levels measured from the fifth day post SAH to the eighth day postSAH were significantly higher in patients developing vasospasm than those who did notdevelop vasospasm, and that CRP values from the third day to the eighth after SAH were obviously higher in patients with a poor outcome than in those with a good outcome [8].

\section{Subjects and Methods}

Study design:

Cross sectional study with stratified random sample of 30 patients, which was designed to test the correlation between level of CRP and incidence of vasospasm in spontaneous $\mathrm{SAH}$, and being an independent predictor for prognosis in patient who had been admitted in Intensive Care Unit (ICU) Neurosurgery Department, Emergency and Trauma Unit at Kasr Al-Ainy, Cairo University Hospitals.

\section{Study patients:}

- Patients who experienced spontaneous subarachnoid hemorrhage from September 2018 to September 2019.

- Screening for CRP was performed to all.

\section{Sample size:}

- Epi-calc 2000 was used to calculate the sample size of this cross sectional study. Assuming $80 \%$ power, 0.05 level of significance, $20 \%$ null hypothesis value and estimated proportion of $10 \%$.

- Assuming 80\% power, 0.05 level of significance, $20 \%$ null hypothesis value and estimated proportion of $10 \%$.

- The sample size used is 30 patients.

\section{Inclusion criteria:}

1- Patients presenting with Spontaneous Subarachnoid Hemorrhage (SAH) confirmed with non contrast CT brain.

2- Age more than 18 years.

3- Both gender.

4- CT angiography or four vessels angiography revealed Aneurysm or AVM as a cause for Spontaneous Arachnoid Hemorrhage (SAH) or patients has clinical vasospasm with no identifiable cause in the angiography.

\section{Exclusion criteria:}

1- Traumatic cases of Subarachnoid hemorrhage.

2- Age less than 18 years.

3- Patients with spontaneous SAH with evident source of infection e.g. chest infection, positive culture, urinary tract infection, wound infection or infective endocardities.

\section{Clinical assesment:}

- History taking: (Sudden onset of a severe headache (often described as "the worst headache of my life"), nausea and vomiting, seizures).

- General examination: (Any signs of infection, ischemic cardiac manifestation, arterial blood gases, central venous pressure).

- Neurlogical examination: (Stiff neck, sensitivity to light (photophobia),blurred or double vision, Hunt and Hess score, loss of consciousness, motor or sensory affection).

\section{Investigations:}

\section{1- CT brain:}

- Evidence of Subarachnoid haemorrhage.

- Presence of infarction or signs of delayed cerebral ischemia.

- Modified Fischer scale.

- Grade 0: No Subarachnoid Hemorrhage (SAH), no Intraventricular Hemorrhage (IVH).

- Grade 1: Focal or diffuse, thin SAH. no IVH.

- Grade 2: Thin focal or diffuse SAH, IVH.

- Grade 3: Thick focal or diffuse SAH.

- Grade 4: Thick focal or diffuse SAH, IVH present.

(Note: Thin SAH is $<1 \mathrm{~mm}$ thick and thick $\mathrm{SAH}$ is $>1 \mathrm{~mm}$ in depth) [10]. 


\section{Vessels or CT angiography:}

- Aneurysm or AVM (site, size, location, fundus direction, neck size).

- Radiological evidence of vasospasm as areas of infarction or stenotic segment.

\section{CRP level:}

- Onday of admission.

- On the 3 rd day.

- Day after day in patients developed vasospasm (clinically or radiologically).

Labs:

- Haematocrit.

- Sodium and potassium level.

- Assessing the level of CRP and correlation with clinical vasospasm, outcome of SAH.

\section{Primary outcomes:}

- Serum CRP as a predictor of vasospasm.

- The rising level of serum CRP as a predictor for poor outcome, morbidity and mortality.

\section{Secondary outcomes:}

- Correlation of vasospasm in patients with AVM.

- Negative angiography after spont SAH and it's correlation with presence of underlying pathology.

- HTN as a morbid risk of patients witSAH.

\section{Results}

The CRP levels in serum peaked on the 3 rd admission day. Patients with lower intial GCS scores and higher Hunt and Hess (grade 4 and 5) and Fisher grades (grade 3 and 4) had statistically significantly higher levels of CRP in serum. Patients with angiographic vasospasm had higher CRP measurements in serum between (96-390). Patients with higher GCS and favourable outcome (glascow outcome scale more than 2) had a lower CRP in the serum (less than 50). Additionally, patients with higher CRP levels in serum (above 100) had less favorable outcome in this study.

\section{Demographics:}

Out of 30 patients included in the study 19 patients $(63.3 \%)$ were males and 11 patients $(36.7 . \%)$ were females.

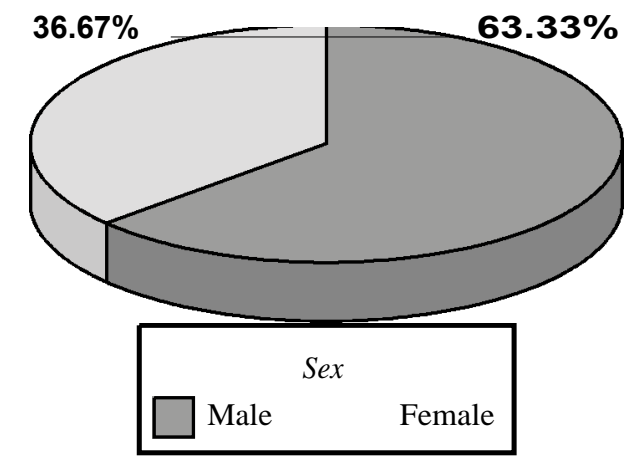

Fig. (1): Distribution of cases according to gender.

Age:

Overall age ranged from 21 years to 80 years with a mean age 53 years.

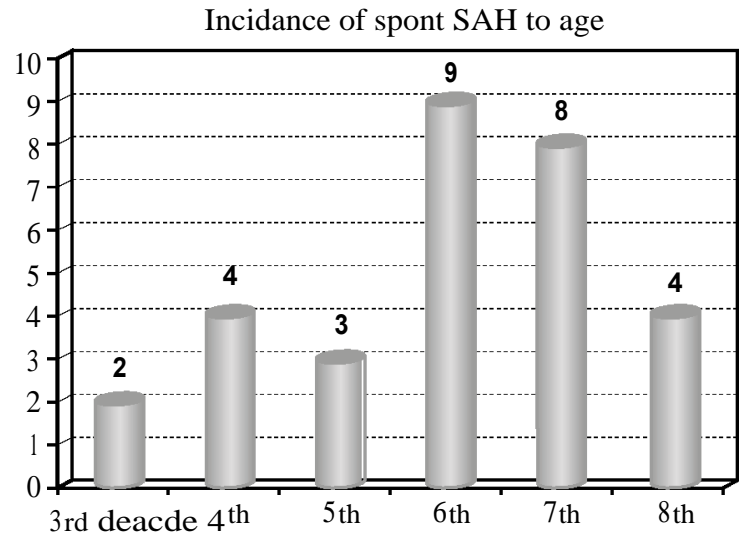

Fig. (2): Incidence of spontaneous subarachnoid hemorrhage accourding to age.

Table (1): Distribution of the studied cases according to age.

\begin{tabular}{cccccccc}
\hline & Mean & Median & Minimum & Maximum & Standard Deviation & Percentile 25 & Percentile 75 \\
\hline Age & 53 & 56 & 21 & 80 & 16 & 42 & 64 \\
\hline
\end{tabular}

\section{Etiology of SAH:}

Out of 30 patients, 16 patients $(53.3 \%)$ had aneurysmal SAH, (10 patients has a.comaneurysm, two patients have basilar tip aneurysm, one patient has MCAaneurysm, one patient has p.com aneurysm, two patients has posterior cerebral aneurysm, three patients $(10 \%)$ of them has subarachnoid from AVM, 11 patients (36.6\%) of them has negative angiography. Out 14 patients who had vasospasm, 10 pateints of them have aneurismal $\mathrm{SAH}$, four patients of them have negative angiography. 


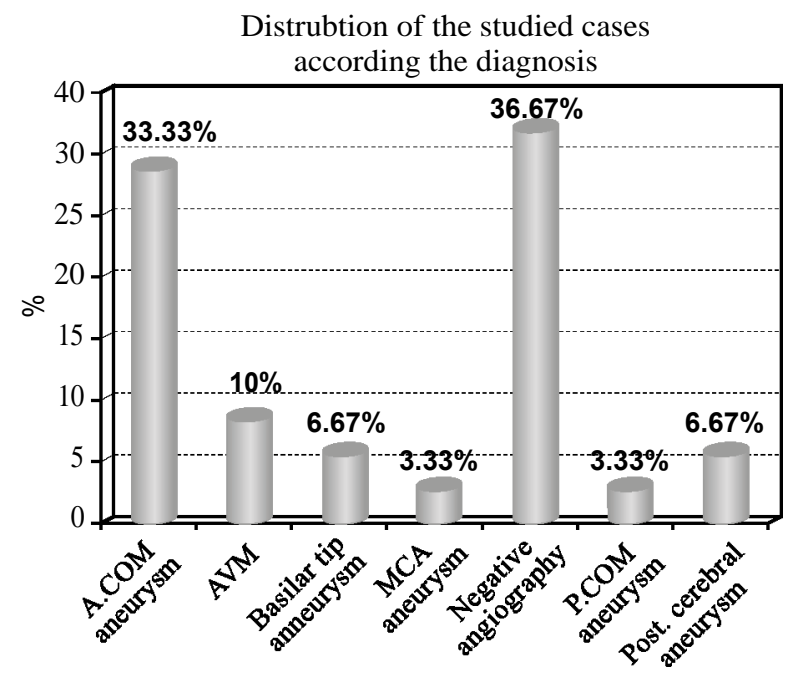

Fig. (3): Distribution of the studied cases according to diagnosis.

\section{Clinical situation:}

Out of 14 patients that had vasospasm, 7 patients $(50 \%)$ primary presented by fits, then 10 patient developed disturbance of conscious level, three of them had motor deficit and one of them had aphasia.

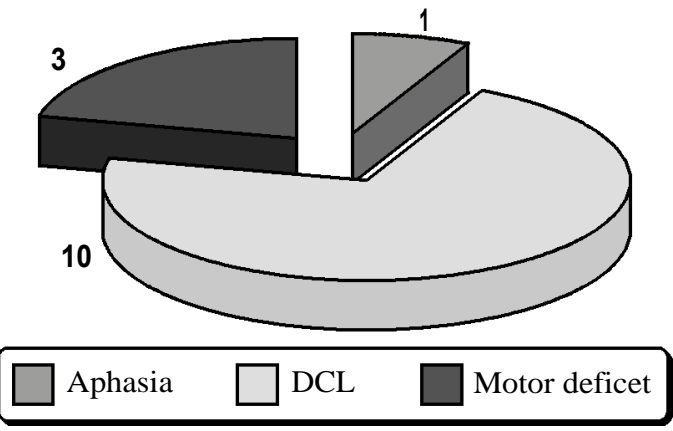

Fig. (4): Distribution of vasospasm cases according to clinical finding.

\section{Radiological finding:}

Out of 30 patients in our study,initially 20 patient has only subarachnoid hemorrhage, four has interventricular hemorrhage, three with intercerebral hematoma, three had gyrus rectus hematoma with midline shift.

Out of 14 patients who developed vasospam, six patients had a.com aneurysm, three patients had negative angiography, two patients had p.com aneurysm, one patient had PCA aneurysm, one patient had basilar tip aneurysm, one patient had MCA aneurysm).

Out of those 14 patient, later on seven patients developed ischemic area in CT, three patients showed vasospasm in cerebral vessels by angiography, four pateints deteriorated clinically without any radiological findings in CT, and couldn't arrange to do 4 vessels angiography due to being unstable, then died.
The rest of 16 patients who didn't experienced vasospasm (eight patients had negative angiography with hydrocepahlic changes in one of them, three patient had AVM with hydrocephalic changes in one of them, four patients had a.com aneurysm, one had PCA aneurysm).

\section{Management:}

Out of 16 pateints that had aneurysmal SAH, nine underwent clipping (six patients of them were a.com aneurysm, two patient of them were MCA, one of them was PCA), six patients underwent coiling (three were a.com aneurysm, two were basilar tip aneurysm, one was PCA aneurysm), one died before any intervention.

Two patients had AVM that was excised, and one patient died after insertion of V-P shunt.

Out of 11 patient that had negative angiography, one of them had hydrocephalic changes and underwent V-P shunt.

Distribution of studied cases according to the management

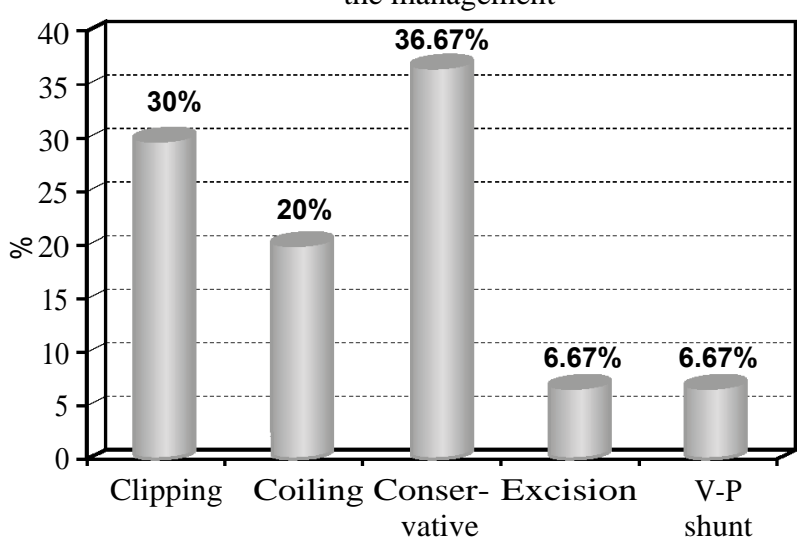

Fig. (5): Distribution of the studied cases according to mangment.

\section{CRP and prognosis:}

Out of 14 patients who developed vasospasm, 13 patients (92\%) initially had high serum CRP level above $50 \mathrm{mg} / \mathrm{L}$, and all patient who had rising CRP level (double to triple folds) had poor outcome.

Out of 16 patients who didn't develop vasospasm, $14(87.5 \%)$ patients initially had low serum CRP level below $50 \mathrm{mg} / \mathrm{L}$, only two patients had high level which decreased on the 3 rd day to normal levels (below $6 \mathrm{mg} / \mathrm{L}$ ).

\section{Risk factors:}

Out of 30 patients included in study, 17 patients (56.6\%) had medical history of HTN, nine patients of them were among group who developed vasospasm. 
Table (2): Distribution of the studied cases according to CL and CRP level.

\begin{tabular}{lllllclc}
\hline & Mean & Median & Minimum & Maximum & Standard Deviation & Percentile 25 & Percentile 75 \\
\hline CL on admission/15 & 13 & 14 & 5 & 15 & 3 & 11 & 15 \\
CRP on admission & 41.7 & 21.0 & 4.0 & 180.0 & 45.7 & 10.0 & 66.0 \\
Follow-up CRP & 115 & 43 & 5 & 390 & 121 & 12 & 210 \\
\hline
\end{tabular}

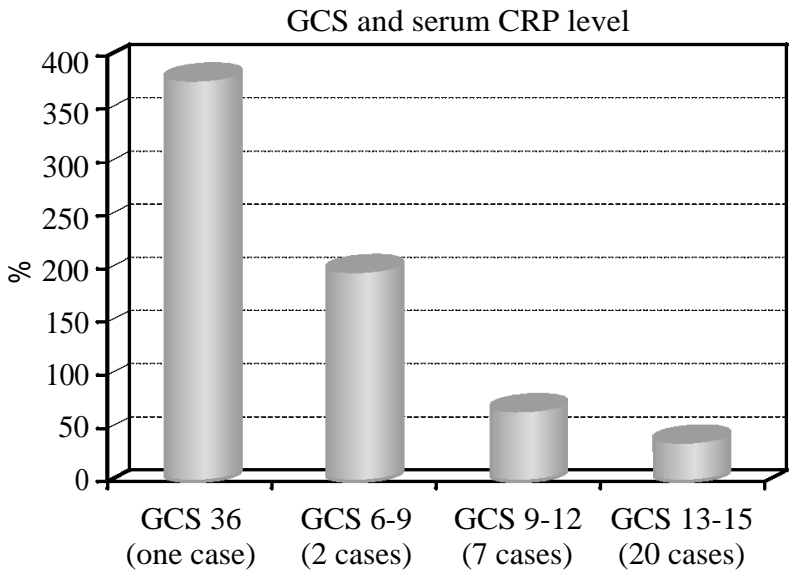

Fig. (6): Correlation of the studied cases according to CL and CRP level, number on the columns demonstrate number of patients in each group.

Table (3): $p$-value of the correlection between vasospasm, poor outcome and CRP level.

\begin{tabular}{|c|c|c|c|c|}
\hline Vasospasm & $\begin{array}{c}\text { CRP } \\
\text { on admission }\end{array}$ & $\begin{array}{c}p- \\
\text { value }\end{array}$ & $\begin{array}{l}\text { Follow-up } \\
\text { CRP }\end{array}$ & $\begin{array}{c}p- \\
\text { value }\end{array}$ \\
\hline \multicolumn{5}{|l|}{$\begin{array}{l}\text { No: } \\
\bullet N:\end{array}$} \\
\hline $\begin{array}{l}\text { Valid } \\
\text { Missing }\end{array}$ & 16 & 0.007 & 16 & $<0.001$ \\
\hline - Median & 13.000 & & 12.50 & \\
\hline $\begin{array}{l}\text { - Percentiles: } \\
25 \\
75\end{array}$ & $\begin{array}{l}7.500 \\
21.500\end{array}$ & & $\begin{array}{l}7.25 \\
21.00\end{array}$ & \\
\hline $\begin{array}{l}\text { Yes: } \\
\cdot N:\end{array}$ & & & & \\
\hline $\begin{array}{l}\text { Valid } \\
\text { Missing }\end{array}$ & $\begin{array}{l}14 \\
0\end{array}$ & & $\begin{array}{l}14 \\
0\end{array}$ & \\
\hline - Median & 49.500 & & 210.50 & \\
\hline $\begin{array}{l}\text { - Percentiles: } \\
25 \\
75\end{array}$ & $\begin{array}{l}22.250 \\
116.750\end{array}$ & & $\begin{array}{l}157.75 \\
295.00\end{array}$ & \\
\hline
\end{tabular}

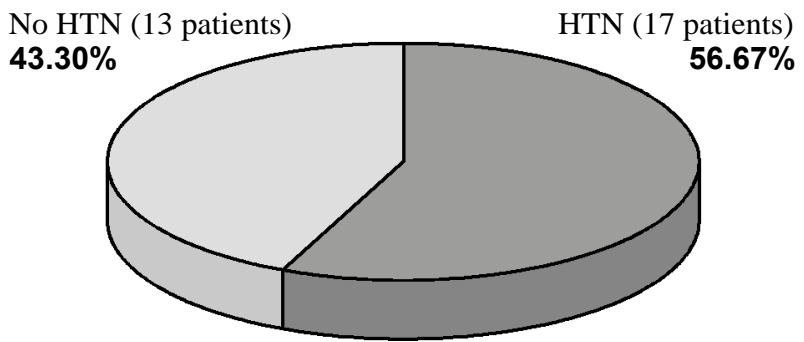

Fig. (7): Correlation of HTN and spontaneous SAH.

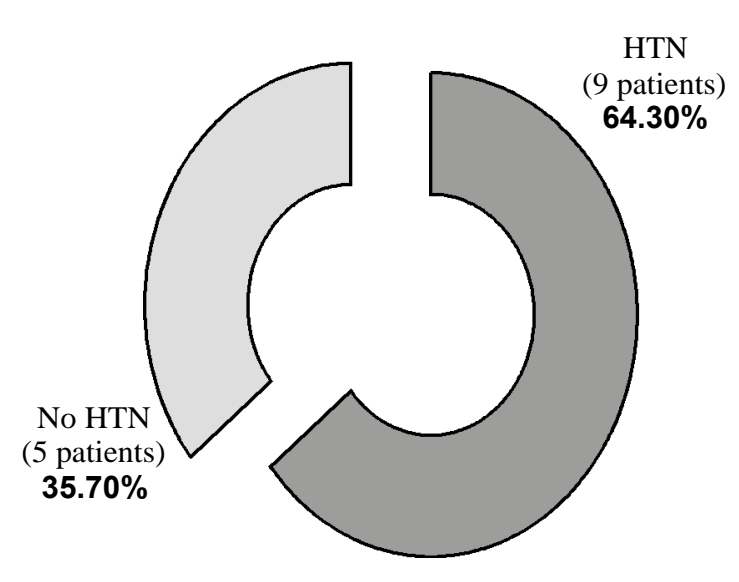

Fig. (8): Correlation of HTN to occurance of vasospasm.

\section{Discussion}

Cerebral vasospasm post-SAH is common and potentially devastating, yet still incompletely understood. Delayed cerebral vasospasm has been correlated with high rates of morbidity and mortality [11].

Although the potential role of inflammation in the genesis of cerebral vasospasm has been recognized for some time, its molecular and cellular basis and its putative importance have not been more clearly defined until recently [12]

CRP is a sensitive inflammatory marker, where its synthesis in hepatocytes is strongly stimulated by interleukin-6 [13]. Moreover, IL-1, which has been implicated in the pathogenesis of cerebral vasospasm, also represents a potent stimulus for CRP synthesis [9]. So, elevated concentrations of CRP is also associated with an increased possibility of developing cerebral vasospasm and consequently developing Delayed Ischemic Neurological Deficit (DIND) [14] .

There was a strong inverse correlation between admitting GCS scores and serum levels of CRP, meanwhile Hunt and Hess and Fisher grades were also correlated in a statistically significant fashion with the CRP measurements in our cohort. These data clearly indicates levels of CRP is significantly correlated with the severity of aSAH and development of vasospasm. Furthermore, the elevated CRP 
levels were associated with worse clinical outcome, as expressed in GCS.

In ourstudy, the strict inclusion criteria had mimimized the influence of other confounding factors such as concomitant systemic conditions or systemic infection, and the statistical analysis is compelling to define the impact of CRP levels on the occurrence of vasospasm and neurological final outcomes.

It is well known that clinical outcome in patients with anurysmal SAH is multifactorial. The association between CRP levels systemically with the clinical outcome could be influenced by other parameters in a complex and usually unpredictable way. In addition, CRP represents a sensitive but also a nonspecific inflammatory marker [13].

Our study was conducted prospectively upon 30 patients presenting with spontaneous subarachnoid hemorrhage. $63.3 \%$ of them were males, $36.7 \%$ were female with mean age was 53 .

Male predominance of our study is agreed with the studyof Fountas et al., [9] in which 41 patients were studied, $61 \%$ were men and $39 \%$ were female with mean age 51.8 .

Our study disagreed with the study of Juvela et al., [14] which $50.5 \%$ were female, $94.5 \%$ were males with mean age 50.3 and disagreed with the study of Rothoerl et al., [8] which 88 patients were prospectively studied, male percentage $38.6 \%$, female percentage $61.4 \%$ with mean age 52.5.

In our study, we detected a diverse causes of spontaneous subarachnoid hemorrhage, out of 30 patients, 16 patients $(53.3 \%)$ had aneurysmal SAH (ten patients has anterior communicating (A.com) artery aneurysm, two patient has basilar tip aneurysm, one patient has Middle Cerebralartery (MCA) aneurysm, one patient has posterior communicating (P.com) artery aneurysm, two patients has posterior cerebral aneurysm), three patient (10\%) of them has subarachnoid from AVM, eleven (36.6\%) of them has negative angiography.

Which agreed that A.com aneurysm is the most common cause of spontaneous SAH going with the study of Rothoerl et al., [8] in which all cases were aneurysmal subarachnoid hemorrhage, 40 patients has ACom, 21 patient has MCA, 7 patient has P.com, 2 patients has posterior cerebral, 17 patients has Internal Carotid Artery (ICA). And agreed with the study of Fountas, et al., [9] in which all cases were aneurysmal subarachnoid hemorrhage, 11 of patients has A.com, 5 patient has basilar, 7 patient has MCA, 14 patient has P.com , 2 patients has posterior inferior cerebellar, 5 patients has ICA, 2 of the patient has anterior choroidal artery).

Out of 30 patient included in our study 17 patient had medical history of HTN, and out of 14 patients who experienced vasospasm, 9 of them had medical history of HTN (64.2\%).

And accourding to Jeon et al., [15], in which out of 93 patients included in the study, 47 (50.5\%) patients had history of HTN, and out of 26 patients who had vasospasm 11 of them has HTN.

The two studies agreed that HTN is a risk factor for aSAH but disagreed in correlation between vasospasm and HTN.

In our study, out of 14 patients who developed vasospam, 7 patients had ischemic area in CT, 3 patients showed vasospasm in cerebral vessels by 4 vessels angiography, 4 pateints deteriorated clinically without any radiological findings. According to Rothoerl et al., [8] all of the patients in his study showed delayed ischemic lesions in the CT scan. In the study of Juvela et al., [14] the causes of poor clinical condition were determined by routine repeated CT scanning, post-operative angiograms, or laboratory investigations.

In our study, out of 16 pateint who had aSAH, 9 underwent clipping, 6 coiling, one died before any intervention with main mangament is open surgery, which agreed with Juvela et al., [14] which out of 178 of his patient the ruptured aneurysms were occluded by open surgery (173 patients) or by endovascular coiling (five patients). And agreed with Rothoerl et al., [8] which all patient underwent open surgery because endovascular treatment was an exclusion criteria in this study.

According to Badjatia et al., [16] out of 110 patient included in his study, 74 patient underwent clipping and 36 patient underwent coiling, which is agreed to our study with the predomincane of clipping in the treatment of cerebral aneurysm.

In our study out of 30 patients who had spontaneous SAH, 13 patient had poor outcome $(43.33 \%)$ and 17 patients discharged (56.67\%) but half of them with morbidities like aphasia or motor deficit with mild improvement after follow-up in our outpatient clinic after 3 months, in comparison to Jeon et al., [15] out of 93 patient included in the study 70 patients had good outcome while 23 had poor outcome and accourding Juvela et al., [14] 
$69.6 \%$ of patient had favourable outcome while $30.4 \%$ \& had poor outcome.

In our study, out of 30 patient included, 14 patient (46.\%), experienced vasospasm, out of this 14 patients, 13 patients $(92 \%)$ initially had high serum CRP level above $50 \mathrm{mg} / \mathrm{L}$, with $p$-value 0.007 , and by following-up serum level CRP in those patients, we found that all patient who had rising CRP level (double to triple folds) had poor outcome with $p$-value $>0.001$.

Which also was found by Jeon et al., [15] were (30\%) of patients showed post-operative symptomatic vasospasm during the hospital course, and CRP was an independent factor in predicting vasospasm, both pre-operative and post-operative CRP levels were significantly higher in patients with an unfavorable outcome than in patients with a favorable outcome, and the post-operative CRP rather than the pre-operative CRP was a useful prognostic factor for poor outcome in patients with aneurysmal SAH; with $p<0.01$.

And agreed with Fountas et al., [9] whose results showed that elevated CRP levels in serum and CSF were associated with increased incidence of angiographic vasospasm with $p<0.0001$ and no statistically significant difference in the serum CRP levels between the group of patients undergoing surgical clipping and those undergoing endovascular coiling.

And according to Ralf Rothoerl et al., [8]. We could confirm this increase of serum CRP levels after acute aneurysmal SAH especially after day 3 , there is a statistically significantly higher increase in patients developing DIND in the later time course with $p<0.025$.

Juvela et al., [14] found that CRP values in those with poor outcome higher than corresponding values in those with favourable outcome with $p<0.001$ and those with open aneurysm surgery had similar CRP values to those with aneurysm coiling (which agreed with our study that no statistically significance in CRP value between patients who had coiling and those who had clipping), and elevated CRP level can be used as a marker of a risk factor for poor outcome after SAH, particularly in sedated patients whose clinical condition is difficult to be estimated.

\section{Conclusion:}

- Vasospasm is a serious event complicating spontaneous aneurismal subarachnoid hemorrhage, it could be diagnosed clinically or radiologically.
- Patient who had medical history of hypertention increased risk of spontaneous subarachnoid hemorrhage and vasospasm.

- Close monitoring and proper management can prevent deterioration and avoid poor outcome of vasospasm.

- High serum C-reactive protein level is an indicator of cerebral vasospasm following aneurysmal subarachnoid hemorrhage.

- Rising serum C-reactive protein level is an independent predictor for poor outcome of aneurysmal subarachnoid hemorrhage.

- Serum level of C-reactive protein on admission, third day, then day after day may help to predict early deterioration in cases of spontaneous subarachnoid hemorrhage and further better management.

\section{Conclusion:}

Subarachnoid Hemorrhage (SAH) is a pathologic condition that occurs when blood breaches the subarachnoid space. The most common cause of SAH is head trauma, and aneurysms are the most common cause of spontaneous SAH.

Spontaneous SAH can be diagnosed easily by Computed Tomography, but the underlying pathology of the cause of SAH need more investigation like CT angiography or 4 vessels angiography.

Cerebral vasospasm is recognized sequel of aneurismal subarachnoid hemorrhage which can lead to neurological deterioration or poor outcome following aneurismal rupture.

\section{References}

1- HWANG, SUNG-HWAN, et al.: "Significance of Creactive protein and transcranial Doppler in cerebral vasospasm following aneurysmal subarachnoid hemorrhage." Journal of Korean Neurosurgical Society, 54.4: $289,2013$.

2- KASSELL, NEAL F., et al.: "The International Cooperative Studyon the Timing of Aneurysm Surgery: Part 1: Overall management results." Journal of neurosurgery, 73.1: 1836, 1990.

3- KASSELL N.F., et al.: "Cerebral vasospasm following aneurysmal subarachnoid hemorrhage." Stroke, 16.4: 56272, 1985.

4- DHAR, RAJAT and MICHAEL N. DIRINGER: "The burden of the systemic inflammatory response predicts vasospasm and outcome after subarachnoid hemorrhage." Neurocritical care, 8.3: 404-12, 2008.

5- ZHANG, WENSHENG, et al.: "Clinical significance of changes in IL-6, CRP and S100 in serum and NO in cerebrospinal fluid in subarachnoid hemorrhage and 
prognosis." Experimental and therapeutic medicine, 16.2: 816-20, 2018.

6- BUCKLEY, DAVID I., et al.: "C-reactive protein as a risk factor for coronary heart disease: A systematic review and meta-analyses for the US Preventive Services Task Force." Annals of internal medicine, 151.7: 483-95, 2009.

7- GRAETZ, DANIELA, et al.: "High ICP as trigger of proinflammatory IL-6 cytokine activation in aneurysmal subarachnoid hemorrhage." Neurological research, 32.7: 728-735, 2010.

8- ROTHOERL, RALF DIRK, et al.: "Possible role of the C-reactive protein and white blood cell count in the pathogenesis of cerebral vasospasm following aneurysmal subarachnoid hemorrhage." Journal of neurosurgical anesthesiology, 18.1: 68-72, 2006.

9- FOUNTAS, KOSTAS N., et al.: "Serum and cerebrospinal fluid C-reactive protein levels as predictors of vasospasm in aneurysmal subarachnoid hemorrhage." Neurosurgical focus, 26.5: E22, 2009.

10- HUSSAIN F., RAFAY M., GULZAR F. and SHARIF S.: Traumatic Subarachnoid Hemorrhage Comprising Outcome with Modified Fisher Grade. Surgery Curr. Res., Vol. 9 Iss. 2 No: 330, 2019.

11- BERK, BRADFORD C., WILLIAM S. WEINTRAUB and R. WAYNE ALEXANDER: "Elevation of C-reactive protein in "active" coronary artery disease." The American journal of cardiology, 65.3: 168-72, 1990.

12-ROMERO, FLÁVIORAMALHO, et al.: "Serum C-reactive protein levels predict neurological outcome after aneurysmal subarachnoid hemorrhage." Arquivos de neuropsiquiatria, 70.3: 202-5, 2012.

13- FOUNTAS, KOSTAS N., et al.: "Terson hemorrhage in patients suffering aneurysmal subarachnoid hemorrhage: Predisposing factors and prognostic significance." Journal of neurosurgery, 109.3: 439-44, 2008.

14- JUVELA, SEPPO, KRISTIINA POUSSA, and MATTI PORRAS: "Factors affecting formation and growth of intracranial aneurysms: A long-term follow-up study." Stroke, 32.2: 485-91, 2001.

15-JEON, YOUNG-TAE, et al.: "The post-operative C-reactive protein level can be a useful prognostic factor for poor outcome and symptomatic vasospasm in patients with aneurysmal subarachnoid hemorrhage." Journal of neurosurgical anesthesiology, 24.4: 317-24, 2012.

16- BADJATIA, NEERAJ, et al.: "Relationship between Creactive protein, systemic oxygen consumption, and delayed cerebral ischemia after aneurysmal subarachnoid hemorrhage." Stroke, 42.9: 2436-42, 2011.

\section{قيمة بروتين سى التفاعلى باعتباره مؤشراً للتنبؤ

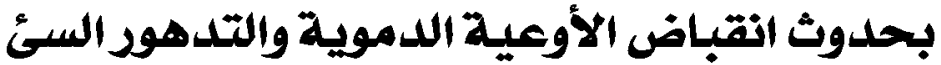 فى حالات النزيف التلقائى تحت غشاء الأدياء الام العنكبوتية}

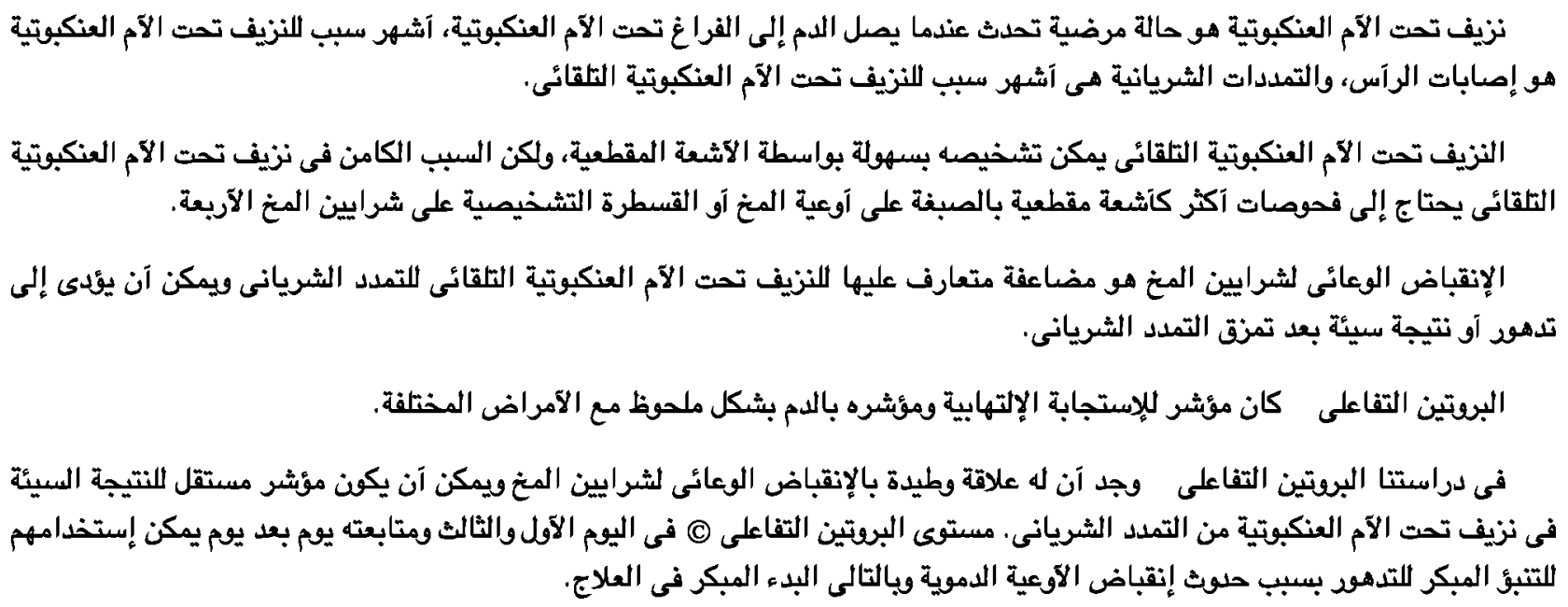

